\title{
La presencia del framing en los artículos publicados en revistas hispanoamericanas de comunicación indexadas en Scopus*
}

\section{Valeriano Piñeiro-Naval' Rafael Mangana ${ }^{2}$}

Recibido: 2017-10-04

Enviado a pares: 2017-10-13
Aprobado por pares: 2017-11-29

Aceptado: 2018-01-21

DOI: 10.5294/pacla.2019.22.1.6

\section{Para citar este artículo / to reference this article / para citar este artigo}

Piñeiro-Naval, V. y Mangana, R. (2019). La presencia del framing en los artículos publicados en revistas hispanoamericanas de comunicación indexadas en Scopus. Palabra Clave 22(1), e2216. DOI: 10.5294/pacla.2019.22.1.6

\section{Resumen}

La importancia de la teoría del encuadre en el dinámico campo de la comunicación resulta indiscutible, ya que ha sido y es una de las más empleadas en la investigación académica. Dada su relevancia, se antoja necesario radiografiar la presencia y el uso que los estudiosos le confieren como herramienta para la comprensión de la realidad mediática. Para acometer este objetivo, se efectuó un análisis de contenido sobre una muestra de 104 artículos publicados en las principales revistas Scopus del área durante la última década en el periodo de 2007 a 2016. A la luz de los resultados, la

\footnotetext{
* Estudiofinanciado porla Fundação para a Ciência e a Tecnologia (FCT) de Portugal(ref.SFRH/BPD/122402/2016) mediante el "Concurso para Atribuição de Bolsas de Doutoramento e Pós-Doutoramento, 2016" (http://www.fct. pt/apoios/bolsas/concursos/individuais2016.phtml.pt)

1 orcid.org/0000-0001-9521-3364. Universidade da Beira Interior, Portugal.vale.naval@labcom.ubi.pt

2 orcid.org/0000-0002-5900-2217. Universidade da Beira Interior, Portugal. rafael.mangana@labcom.ubi.pt
} 
investigación hispanoamericana sobre framing no se aleja de las pautas de producción internacional: gran aplicabilidad empírica, continuismo en los objetos de investigación, conservadurismo metodológico y adquisición de un estatus propio por parte del paradigma.

\section{Palabras clave (fuente: tesauro de la Unesco)}

Investigación sobre la comunicación; teoría del framing; encuadre; revistas científicas; Scopus; análisis de contenido. 


\section{The Presence of Framing in Articles Published in Spanish American Communication Journals Indexed in Scopus}

\section{Abstract}

The importance of the framing theory in the dynamic field of communication is indisputable since it has been and is one of the most used in academic research. Given its relevance, it seems necessary to analyze in depth the presence and use that scholars make of it as a tool to understand media reality. To accomplish this objective, a content analysis was carried out on a sample of 104 articles published in the main Scopus journals in the field in the last decade, for the period between 2007 and 2016. In light of the results, Spanish American research on framing does not depart from international production guidelines: great empirical applicability, continuity in research subject matters, methodological conservatism and the paradigm's acquisition of status.

\section{Keywords (source: Unesco Thesaurus)}

Research; communication research; framing theory; framing; scientific journals; Scopus; content analysis. 


\section{A presença do framing nos artigos publicados em revistas hispano- americanas de comunicação indexadas na Scopus}

\section{Resumo}

A importância da teoria do framing no campo dinâmico da comunicação é indiscutível, uma vez que foi e é uma das mais utilizadas na pesquisa acadêmica. Dada a sua relevância, parece necessário radiografar a presença e o uso que os estudiosos lhe dão como ferramenta para a compreensão da realidade midiática. Para alcançar este objetivo, foi realizada uma análise de conteúdo em uma amostra de 104 artigos publicados nas principais revistas Scopus da área durante a última década, no período de 2007 a 2016 . À luz dos resultados, a pesquisa hispano-americana sobre framing não se afasta das pautas de produção internacional: grande aplicabilidade empírica, continuidade nos objetos de pesquisa, conservadorismo metodológico e aquisição de um status próprio pelo paradigma.

\section{Palavras-chave (fonte: tesauro da Unesco)}

Pesquisa; pesquisa em comunicação; teoria de enquadramento; enquadramento noticioso; revistas científicas; Scopus; análise de conteúdo. 


\section{Introducción y justificación del estudio}

La jerarquía que ostenta la teoría del encuadre en el atomizado campo de las ciencias sociales es indiscutible y, de modo muy especial, en la disciplina que nos ocupa: la comunicación. Referentes internacionales como Bryant y Miron (2004), Van Gorp (2005) o Weaver (2007) sostienen que esta teoría se ha erigido en una de las más divulgadas tanto en las últimas décadas del siglo XX como en los primeros años del siglo XXI. Naturalmente, esta circunstancia la ha aupado a un lugar prominente en el aparataje epistemológico de la investigación mediática.

Vliegenthart y Van Zoonen (2011) articulan esta idea de una forma muy sencilla al manifestar que framing es, sin duda, una de las palabras más de moda en la actualidad. De hecho, si introducimos este término anglosajón en el buscador especializado Google Scholar, obtendremos cerca de 1,7 millones de resultados; ${ }^{3}$ una cifra que representa todo un maremágnum de documentos versados sobre la temática. Por esta razón, en los últimos tiempos, "se puede percibir un constante aumento de los estudios que recurren a los encuadres como una herramienta conceptual básica" (Vicente-Mariño y López-Rabadán, 2009, p. 15).

Hallahan (1999) reconoce que la teoría del encuadre ha sido utilizada para entender e investigar la comunicación desde una amplia variedad de disciplinas, entre ellas, la psicología, la comunicación discursiva, la toma de decisiones en organizaciones, la economía, la comunicación para la salud y la comunicación política. Reese (2001), por su parte, determina que "el concepto de encuadre se ha hecho cada vez más atractivo para la investigación de medios, encontrando su camino en una serie de campos relacionados" (p. 7), entre ellos, a juicio del autor, la sociología y la ciencia política. Asimismo, Hart (2011) esgrime que "el framing ha servido como un robusto y amplio cuerpo teórico que ha sido utilizado en múltiples ramas de la literatura, la investigación y las disciplinas académicas" (p. 31).

3 Dato consultado en: 07-09-2018. 
En suma, es conveniente subrayar, una vez más, la importancia y la permeabilidad del concepto de encuadre en el ecléctico terreno de las ciencias sociales, una relevancia que justifica el presente estudio, cuyo objetivo primigenio radica en cartografiar la presencia y los usos conferidos al framing en los artículos publicados en las principales revistas hispanoamericanas de comunicación indexadas en Scopus, todos ellos comprendidos en el periodo de 2007 a 2016, a saber, en la última década.

\section{Evolución cronológica del framing}

Según McCombs y Ghanem (2001), "los orígenes del término encuadre en el contexto de la comunicación se remontan a la fotografía y la cinematografía, donde encuadrar se refiere a variables tales como el ángulo de la cámara y la perspectiva en el estilo de un mensaje visual" (p. 71). No obstante, tal y como se concibe en esta investigación, "encuadrar es seleccionar aspectos de una realidad percibida y hacerlos más relevantes" (Canel, 1999, p. 101) en el texto informativo.

De manera consensuada, y como se avanzaba en la introducción, se ha advertido que el concepto de encuadre presenta un marcado carácter multidisciplinar. Gran parte de la responsabilidad de este cariz se debe a sus orígenes y ámbitos de aplicación tan diversos. Siguiendo de nuevo a Bryant y Miron (2004), Giménez-Armentia (2006), Gradim (2016) o Van Gorp (2007), se puede constatar que el término surge en el campo de la psicología cognitiva de la mano de Bateson (1955), quien lo empleó para explicar el modo en que los sujetos conocen el mundo que los rodea a través de su focalización en unos aspectos de la realidad en detrimento de otros, esto es, mediante procesos cognitivos individuales.

Posteriormente, el framing es adoptado por otras disciplinas, a menudo con un cambio sutil en su significado, entre ellas, la sociología. En este sentido, el investigador que dota de una dimensión sociológica al concepto de encuadre es Goffman (1974), que señala que lo esencial no es la realidad sino cómo esta es interpretada y valorada por los individuos, tras lo cual se produce una interacción entre los marcos sociales y los esquemas mentales de cada sujeto. 
Ya en el terreno de la información, el desarrollo del framing "es bastante notable, sobre todo porque entronca con uno de los debates sempiternos referido a los medios de comunicación: la posibilidad del periodista de transmitir la realidad tal como es" (Sádaba, 2001, p. 156). Podemos establecer la evolución cronológica del paradigma gracias a la inmediata identificación de tres periodos claramente diferenciados: una primera etapa de desarrollo teórico, una segunda basada en su reorganización y, finalmente, una tercera en la que prevalece su aplicabilidad empírica. Lo que no resulta tan sencillo es acotar con exactitud las distintas fases. Vicente-Mariño y López-Rabadán (2009) aseguran que "es posible estructurar los estudios de framing dentro de una línea de trabajo heterogénea, pero constante desde hace décadas, $y$ dividirla en tres grandes etapas: una fase inicial de formación (1974-1990), una segunda época de definición como especialidad de estudio mediático y de aplicación descontrolada (1991-1999) y, finalmente, la etapa actual de reorganización teórica y desarrollo empírico (2000-)” (p. 17).

Por su parte, Saperas y Carrasco-Campos (2015) no difieren en exceso de lo estipulado por aquellos autores; sin embargo, se guían por la producción científica de la revista Journal of Communication para delimitar estos tres lapsos temporales de distinta forma. En su opinión, la primera etapa se extiende desde comienzos de la década de 1970 hasta 1993, fecha de publicación de un texto nuclear para la comprensión de la teoría que nos ocupa: "Framing: Toward clarification of a fractured paradigm", firmado por Robert M. Entman (1993). Esta fase inicial "destaca por el reconocimiento de los procesos de encuadre como problema comunicativo y por las primeras propuestas de definición teórica a partir de ciencias sociales consolidadas" (Saperas y Carrasco-Campos, 2015, p. 50), entre ellas, como habíamos señalado, la psicología y la sociología.

El segundo de los tramos históricos de desarrollo del paradigma abarcaría la producción científica comprendida entre 1993 y 2007, de nuevo un año en que vio la luz otro trabajo teórico indispensable a cargo, esta vez, de Dietram A. Scheufele y David Tewksbury (2007), "Framing, agendasetting, and priming: The evolution of three media effects models". Esta aportación y otras semejantes integraban un número especial de la revista 
abanderada de la International Communication Association que, en su conjunto, se planteaba como objetivo abordar las grandes disyuntivas existentes hasta la fecha, entras ellas, la consideración del paradigma fracturado (Entman, 1993) frente a la visión multiparadigmática (D’Angelo, 2002) o el estatus independiente de la teoría frente a "su hermana mayor", la agenda-setting (McCombs y Valenzuela, 2007). Así pues, y a juicio de Saperas y Carrasco-Campos (2015), este ciclo "viene marcado por la necesidad consciente de aclarar el lugar de la noción de framing dentro del espacio propio de investigación en comunicación" (p. 50). Finalmente, y

\begin{abstract}
tras una primera fase de préstamo de la noción de framing desde la sociología y la psicología y una segunda fase de fuerte debate teórico-metodológico en torno a la disputa operativa y conceptual del framing, el especial publicado en Journal of Communication abriría un tercer periodo en el que destacaría el estatus del framing como programa empírico ampliamente dominante (Saperas y CarrascoCampos, 2015, p. 51).
\end{abstract}

Las coordenadas en que nos encontramos se caracterizan, pues, por una notable preeminencia del componente práctico y aplicado del paradigma en detrimento de reflexiones teóricas y debates metodológicos, un hecho que nos permite plantear la primera de las hipótesis de nuestro estudio:

- H1: la reciente investigación hispanoamericana (periodo de 2007 a 2016) basada en la teoría del framing tendrá un marcado carácter empírico.

Habiendo efectuado un breve repaso acerca de la evolución cronológica de la teoría del encuadre, en el siguiente apartado, se aludirá a distintos estudios que, al igual que el presente, han radiografiado el estado de la investigación internacional sobre framing con arreglo a distintos parámetros analíticos.

\title{
El estado de la investigación internacional reciente sobre framing
}

Como se ha constatado, la teoría del encuadre es uno de los paradigmas de mayor recorrido y éxito en la investigación en comunicación. Ante esta 
privilegiada posición, estimulada desde 2007 por la abundancia de estudios empíricos sobre la materia, han surgido varias iniciativas centradas en organizar y sistematizar la producción científica versada en el framing. Tal es el caso de Matthes (2009), cuyo trabajo se basa, desde el punto de vista metodológico, en un análisis de contenido de las más importantes revistas internacionales sobre comunicación, en el que dispone de una muestra de 131 artículos publicados entre 1990 y 2005 . Su propósito era claro: evaluar los artículos sobre framing para poder hacer un perfil genérico de cómo se empleaba esta teoría en la investigación mediática. Principalmente, Matthes concluye que "los investigadores sobre framing necesitan reflexionar de manera crítica acerca de cinco puntos clave que les permitan hacer avanzar a esta fructífera área de investigación” (2009, p. 359). Dichos puntos son los que siguen:

- En gran parte de la literatura, las definiciones sobre framing no se trasladan de manera transparente en pasos concretos y operativos.

- Existe una clara división conceptual entre los estudios sobre encuadres específicos y encuadres genéricos.

- La mayor parte de los estudios son descriptivos, pues no contrastan hipótesis relacionadas con la teoría del encuadre. Esto es aún más evidente cuando se trata de estudios sobre encuadres específicos. Sin duda, los estudios descriptivos son de un gran valor para el campo de la comunicación, y el framing es un concepto muy útil para contextualizar y describir contenidos mediáticos. Sin embargo, este autor asevera que, para progresar en la teoría como un campo esencial de la comunicación, son necesarias menos estrategias descriptivas.

- Por otro lado, no hay demasiadas evidencias acerca de los modos en que los elementos visuales son incorporados a los encuadres.

- Finalmente, la ausencia de informes sobre fiabilidad en gran parte de los estudios es un problema para la investigación sobre framing. De hecho, nada menos que el $48 \%$ de los estudios carecen de información a este respecto.

Ligeramente más cercano en el tiempo, un segundo trabajo destacado es el de Borah (2011), que, al igual que Matthes (2009), efectúa un análisis 
de contenido; en este caso, sobre una muestra de 379 artículos procedentes de 93 revistas científicas. El resultado de su trabajo ofrece una nítida instantánea de la producción sobre framing desde 1997 hasta 2007. Borah incide en la permeabilidad temática del concepto y establece ciertas preguntas de investigación a las que da respuesta a la luz de sus resultados:

- ¿ ¿Qué aproximación al estudio de los encuadres es más recurrente, la sociológica o la psicológica? Es decir, los encuadres mediáticos sociológicos, medidos mediante el análisis de contenido, frente a encuadres individuales psicológicos, medidos con encuesta o experimento. Pues bien, el 61,5\% de los estudios presentan un perfil sociológico a través de la aproximación metodológica del análisis de contenido.

- ¿Qué es más frecuente, el empleo de encuadres específicos ideados para un estudio en concreto o encuadres genéricos extensibles teóricamente a otros estudios? En este sentido, son los específicos los que acaparan un mayor porcentaje: el 49,1\%.

- iSe tiende a emplear el framing como una teoría con entidad propia e independiente? Pues bien, está ganando peso como una teoría al margen de otras con las que determinados autores sí la hayan podido relacionar, como es el caso de la agenda-setting o el priming.

El último de los trabajos analíticos por resaltar es el efectuado por Saperas y Carrasco-Campos (2015), quienes plantean también un análisis de contenido cimentado en la evaluación de una muestra de 50 papers pertenecientes a la revista Journal of Communication, en el periodo 2009 de 2013. El libro de códigos que usaron está estructurado con arreglo a parámetros como el tipo de investigación, los objetos de estudio, las técnicas metodológicas y los procedimientos de construcción teórica. Tras su examen, los autores llegan a la conclusión de que existe "cierto conservadurismo en los objetos y temas investigados, una propuesta casi unidireccional en el desarrollo metodológico, y cierta modestia en los diseños muestrales, poco ambiciosos y sin pretensión de representatividad" (Saperas y Carrasco-Campos, 2015, pp. 63-64).

Obsérvese, por ende, que existen varias carencias en la investigación científica sobre la teoría del encuadre, tanto a nivel teórico como metodo- 
lógico; unas carencias que convendría paliar progresivamente para conferir a la disciplina una mayor calidad y solidez.

Asimismo, los estudios consignados nos posibilitan plantear las restantes hipótesis de investigación relativas a nuestro objeto de estudio, o sea, la reciente investigación hispanoamericana sobre framing:

- $\quad H 2$ : se producirá un notable continuismo y una marcada uniformidad tanto en la selección de los objetos como de los temas de investigación.

- H3: la mayoría de los estudios será de índole inductiva y descriptiva.

- H4: a nivel metodológico, el análisis de contenido se erigirá como un pilar central para el estudio de los encuadres, aunque presentará deficiencias relativas al reporte de la fiabilidad intercodificadores.

- H5: también a nivel metodológico, existirá una relativa modestia en el planteamiento de los diseños muestrales, restringidos más bien al tratamiento de muestras no representativas.

- H6: no abundarán los estudios sobre encuadres visuales.

- H7: la perspectiva mediático-sociológica dominará los trabajos en relación con la individual-psicológica.

- H8: los encuadres específicos superarán en presencia a los genéricos.

- H9: la teoría del framing se habrá ganado un estatus y una entidad propios frente a otros paradigmas, principalmente la agenda-setting.

De inmediato, se describe la metodología que nos ha permitido abordar las hipótesis planteadas.

\section{Metodología}

En línea con los estudios citados, la metodología empleada para el acometimiento del presente trabajo se ha fundamentado en el análisis de contenido. Krippendorff (1990), a colación del proceso de diseño muestral, afirma que "está orientado por un plan de muestreo que especifica con suficiente detalle de qué manera ha de proceder el investigador para obtener una muestra de unidades que en su conjunto sean representativas de la 
población que le interesa” (p. 95). Por tanto, en un primer estadio del proceso, se identificaron diez revistas hispanoamericanas que ejercieron como unidades de recogida de datos. Estas publicaciones, presentes en Scopus en la categoría de communication y ordenadas según su factor de impacto (SJR2016), son las siguientes: Comunicar: Revista Científica de Comunicación y Educación, Revista Latina de Comunicación Social, El Profesional de la Información, Palabra Clave, Communication \& Society, Estudios sobre el Mensaje Periodístico, Cuadernos.info, Historia y Comunicación Social, Comunicación y Sociedad (México) y Scire: Representación y Organización del Conocimiento.

El universo de artículos publicados por estas revistas en el lapso temporal analizado fue de 4095 papers (con excepción de editoriales y reseñas), de los cuales el 2,54 \%, a saber, $\mathrm{n}=104$ artículos aluden, de forma más o menos explícita, a la teoría del encuadre.

La estrategia seguida para la detección de la muestra se basó en la inclusión de los términos framing, frame, encuadre, teoría del framing y teoría del encuadre en los motores de búsqueda internos de los sitios web de las revistas. Aunque el rastreo se centraba, principalmente, en el título, el resumen y las palabras clave de cada paper, los buscadores no solo devolvían resultados con los términos emplazados en las citadas ubicaciones, ya que también figuraban, en ocasiones, a lo largo de los textos. Por ende, se revisaron minuciosamente todos los resultados y se seleccionaron los que, en efecto, tenían ligazón con la teoría del framing.

\section{Categorías de análisis}

Una vez planteadas las hipótesis y escogida la muestra, "se debe elaborar un libro de códigos (codebook) en el que se especifique con gran detalle cómo debe evaluarse cada criterio o variable" (Igartua, 2006, p. 204). Las categorías que han permitido efectuar el examen de los artículos, todas ellas variables de medida nominal politómica y basadas, en su mayoría, en el criterio apriorístico de autores contrastados en la materia, son las siguientes:

- $\quad$ Objeto en el que se centra la investigación, ya sea un medio de comunicación, un soporte documental o, incluso, los propios receptores de la información. Esta variable podía adoptar los siguientes 
valores: 1 = prensa tradicional; $2=$ prensa en línea; $3=$ revistas, suplementos; $4=$ televisión; $5=$ radio, $6=$ cine; $7=$ internet, TIC; $8=$ redes sociales, elementos web $2.0 ; 9=$ spots o soportes publicitarios; 10 = videojuegos; 11 = artículos científicos, investigación académica; 12 = receptores de la comunicación; 13 = otros objetos; y $14=$ varios objetos a la vez.

- Temática del estudio, cuyas opciones de respuesta son las siguientes: 1 = cobertura informativa en general, temas variados; 2 = política, campañas electorales, movimientos de activismo político; $3=$ movimientos sociales, activismo cívico; 4 = sanidad, salud; 5 = economía, finanzas; 6 = conflictos bélicos, procesos de paz, terrorismo, violencia; 7 = fenómenos migratorios, multiculturalismo, minorías étnicas; $8=$ comunicación estratégica, corporativa; $9=$ contenidos persuasivos, publicitarios; 10 = ecologismo, medio ambiente; 11 = cuestiones de género; 12 = estado de la investigación científica; 13 = otras temáticas.

- Modalidad del estudio (Martínez-Nicolás y Saperas, 2016), en la que se contempla: 1 = empírico (se analiza alguna realidad fenoménica relacionada con el framing); 2 = teórico-conceptual (se exponen y discuten teorías y conceptos o se elaboran estados de la cuestión); 3 = metodológico (propuestas sobre métodos, técnicas o procedimientos de investigación); 4 = mixto, combinando distintas modalidades; 9 = no aplicable.

- $\quad$ Tipo de estudio (De Vreese, 2005; Semetko y Valkenburg, 2000), pudiendo ser los siguientes: 1 = inductivo, descriptivo (los encuadres emergen del material durante el curso del análisis); 2 = deductivo, explicativo (son definidos y operacionalizados en investigaciones previas); 3 = ambos; $9=$ no aplicable.

- Técnicas metodológicas empleadas (Saperas y Carrasco-Campos, 2015) que abarcan: 1 = análisis de contenido manual; 2 = análisis de contenido automatizado; 3 = encuesta; 4 = investigación experimental; 5 = análisis del discurso; 6 = entrevistas a profundidad; 7 = grupos focalizados; $8=$ metaanálisis; $9=$ análisis bibliométrico, cibermétrico; 10 = estudio cualitativo de caso; 11 = varias técnicas a la vez; y 12 = no aplicable. 
- En caso de efectuar un análisis de contenido, ¿se reportan datos relativos a la fiabilidad intercodificadores? Las respuestas son estas: $0=$ no se reportan; 1 = sí se reportan; 9 = no aplicable.

- Muestra de casos o individuos con que cuenta el estudio es: 1 = no probabilística, de conveniencia, no representativa; 2 = probabilística, aleatoria, representativa; o $9=$ no aplicable.

Otras especificidades de los estudios examinadas han sido las subsecuentes:

- $\quad$ Estudio centrado en (Muñiz, Igartua, Otero y De la Fuente, 2008): $1=$ framing textual; 2 = framing visual; $3=$ ambos; $9=$ no aplicable.

- Investigación cuya aproximación es (De Vreese, Peter y Semetko, 2001; Scheufele, 1999): 1 = mediática, sociológica (media frames oframes in communication); 2 = individual, psicológica (frames in thought, audience frames o individual frames); 3 = ambas; $9=$ no aplicable.

- Estudio con encuadres (Entman, Matthes y Pellicano, 2009): 1 = genéricos (generic frames); 2 = específicos (issue-specific frames); $3=\mathrm{am}$ bos; 9 = no aplicable.

- Construcción teórica de la investigación (Borah, 2011; Saperas y Carrasco-Campos, 2015), cuyas opciones son: 1 = teoría única y exclusiva; 2 = teoría principal; 3 = teoría complementaria o secundaria; 4 = teoría con función contextual o de background; 9 = no aplicable.

Todas estas categorías fueron trasladadas, de forma esquemática, a una ficha de codificación en la que se registraron los valores correspondientes a cada unidad de análisis; valores luego digitalizados y tratados estadísticamente en el programa SPSS, versión 24.

\section{Codificación y fiabilidad}

La codificación de los 104 artículos científicos de la muestra se llevó a cabo entre el 4 y el 14 de septiembre de 2017. Para el chequeo de la fiabilidad intercodificadores, se seleccionó, de forma aleatoria, una submuestra del $20 \%$ de unidades de análisis; es decir, $n=21$ papers analizados por dos codificadores independientes. A posteriori, se empleó el índice más 
robusto que existe para calcular el acuerdo en la asignación de valores a las distintas variables: el alfa de Krippendorff, calculada gracias a la macro (KALPHA) para SPSS (Hayes y Krippendorff, 2007). En total, la fiabilidad media de las 11 variables ${ }^{4}$ que integran el libro de códigos fue satisfactoria: $M \alpha_{\mathrm{k}}=0,84(D T=0,10)$.

\section{Resultados}

Esta sección está estructurada en función de las distintas hipótesis planteadas. La primera de ellas, que asevera que la reciente investigación hispanoamericana sobre framing tendrá un marcado carácter empírico $(\mathrm{H} 1)$, que implica el predominio de estudios prácticos $(93,2 \%)$ en detrimento de los teórico-conceptuales $(3,4 \%)$ y lde os metodológicos $(3,4 \%)$, se corrobora.

En cuanto a los objetos y temas de investigación (H2), obsérvense en la tabla 1 los porcentajes relativos a las distintas opciones de respuesta que contemplaba el codebook.

\section{Tabla 1. Objetos y temas de investigación abordados en los artículos}

\begin{tabular}{|l|c|l|c|}
\hline \multicolumn{1}{|c|}{ Objetos } & $\%$ & \multicolumn{1}{c|}{ Temas } & $\%$ \\
\hline Prensa tradicional & 50 & Política, campañas electorales, movimientos de activismo político & 26,9 \\
\hline Televisión & 14,4 & Fenómenos migratorios, multiculturalismo, minorías étnicas & 16,3 \\
\hline Varios objetos a la vez ${ }^{*}$ & 7,7 & Conflictos bélicos, procesos de paz, terrorismo, violencia & 14,4 \\
\hline Artículos científicos, investigación académica & 6,7 & Ecologismo, medio ambiente & 10,6 \\
\hline Redes sociales, elementos web 2.0 & 4,8 & Cobertura informativa en general, temas variados & 5,8 \\
\hline Prensa online & 3,8 & Estado de la investigación científica & 5,8 \\
\hline Radio & 2,9 & Movimientos sociales, activismo cívico & 4,8 \\
\hline Internet, TIC & 2,9 & Cuestiones de género & 4,8 \\
\hline Receptores de la comunicación & 2,9 & Sanidad, salud & 3,8 \\
\hline Otros objetos & 1,9 & Economía, finanzas & 2,9 \\
\hline Revistas, suplementos & 1 & Comunicación estratégica, corporativa & 2 \\
\hline Spots publicitarios & 1 & Contenidos persuasivos, publicitarios & 1,9 \\
\hline Total & $\mathbf{1 0 0}$ & Total & $\mathbf{1 0 0}$ \\
\hline
\end{tabular}

" Cuando se tienen en cuenta varios objetos a la vez ( $n=8$ papers), la prensa tradicional vuelve a estar presente en la mayoría de los casos; concretamente, en 6 de ellos.

Fuente: elaboración propia.

4 En el apéndice final, se pueden ver los datos de fiabilidad de cada una de las variables del codebook. 
Los datos porcentuales de la tabla 1 nos permiten afirmar que, en efecto, se produce un marcado continuismo en la selección de los objetos de investigación, ya que dos de cada tres estudios recurren a medios históricamente arraigados como prensa tradicional y la televisión. En cuanto a los temas, cuatro de ellos acaparan el 68,2 \% del total: política, migración, guerras y medio ambiente. No obstante, no podría ratificarse que la uniformidad sea la nota dominante.

En relación con el tipo de estudio predominante $(\mathrm{H} 3)$, la mayoría de los trabajos son de índole inductiva y descriptiva $(57,7 \%)$ frente a los deductivos y explicativos $(35,6 \%)$.

Desde el punto de vista metodológico, la tabla 2 refleja los valores correspondientes a las distintas técnicas empleadas en las investigaciones empíricas identificadas:

\section{Tabla 2. Técnicas metodológicas empleadas en los artículos empíricos}

\begin{tabular}{|l|c|}
\hline \multicolumn{1}{|c|}{ Técnica metodológica empleada } & $\%$ \\
\hline Análisis de contenido manual & 51 \\
\hline Análisis del discurso & 16,3 \\
\hline Varias técnicas simultáneamente* & 8,7 \\
\hline Estudio cualitativo de caso & 4,8 \\
\hline Investigación experimental & 3,8 \\
\hline Análisis bibliométrico, cibermétrico & 3,8 \\
\hline Encuesta & 1,9 \\
\hline Entrevistas en profundidad & 1,9 \\
\hline Análisis de contenido automatizado & 1 \\
\hline Total & $\mathbf{9 3 , 2}$ \\
\hline
\end{tabular}

*Cuando se emplean varias técnicas simultáneamente $(n=9$ papers), el análisis de contenido está presente en 8 casos, que suelen figurar junto con el análisis del discurso $(n=3)$ y de la encuesta $(n=2)$.

Fuente: elaboración propia.

A tenor de los datos de la tabla 2, podemos afirmar que el análisis de contenido es el pilar metodológico central para el estudio de los encuadres (H4). Con respecto al reporte de la fiabilidad intercodificadores que siempre debe figurar en todo estudio de análisis de contenido, cabe señalar que 
son más los trabajos en los que no se incluye información sobre este aspecto crítico del protocolo $(59,8 \%)$ que los que sí lo tienen en cuenta (40,2\%).

Desde el punto de vista de los diseños muestrales (H5), la mayoría de los estudios se restringen al tratamiento de muestras no representativas del universo del que provienen $(69,2 \%)$.

Por lo que a las especificidades de los estudios se refiere, véanse en la tabla 3 los datos recabados.

Tabla 3. Especificidades de los estudios sobre framing

\begin{tabular}{|c|c|c|c|c|c|}
\hline Prisma & $\mathbf{\%}$ & Aproximación & $\mathbf{\%}$ & Tipos & $\mathbf{\%}$ \\
\hline Framing textual & 51 & Mediático-sociológica & 79,8 & Genéricos & 22,1 \\
\hline Framing visual & 6,7 & Individual-psicológica & 5,8 & Específicos & 54,9 \\
\hline Ambos & 32,7 & Ambas & 4,8 & Ambos & 11,5 \\
\hline No aplicable & 9,6 & No aplicable & 9,6 & No aplicable & 11,5 \\
\hline Total & $\mathbf{1 0 0}$ & Total & $\mathbf{1 0 0}$ & Total & $\mathbf{1 0 0}$ \\
\hline
\end{tabular}

Fuente: elaboración propia.

Los encuadres noticiosos textuales, "aquellos que tradicionalmente se han analizado y que están presentes en los textos de las noticias" ( $\mathrm{Mu}-$ ñiz et al., 2008, p. 33), acaparan todo el protagonismo en más de la mitad de las veces ( $51 \%)$; mientras que los visuales, "que se transmiten a través de las imágenes que acompañan la información” (Muñiz et al., 2008, p. 33), lo hacen el 6,7 \% de los casos (H6).

Relativamente a las aproximaciones llevadas a cabo para el estudio del framing $(\mathrm{H} 7)$, está claro que la mediático-sociológica (79,8\%) hegemoniza, sin la menor discusión, los trabajos. Por otro lado, el planteamiento de encuadres de tipo específico ( $54.9 \%)$, únicamente aplicables a un contexto de estudio concreto, predominan frente a la opción genérica $(22,1 \%)$, mediante la cual los encuadres son permeables a distintos objetos y contextos (H8).

Tomando ahora como punto de partida las técnicas metodológicas empleadas y su relación con las aproximaciones al estudio de los encuadres, resulta interesante comprobar si se produce alguna relación significativa 
entre el análisis de contenido, la encuesta y el experimento, y los acercamientos sociológico y psicológico (Borah, 2011) (tabla 4).

\section{Tabla 4. Relación entre la aproximación efectuada para el estudio del framing y las técnicas metodológicas empleadas ( $\%$ columna)}

\begin{tabular}{|c|c|c|c|}
\hline \multirow{2}{*}{ Aproximación } & \multirow{2}{*}{ \% total } & \multicolumn{2}{|c|}{ Técnicas metodológicas } \\
\cline { 3 - 4 } & 91,2 & Análisis de contenido & Encuesta o experimento \\
\hline Mediático-sociológica & 8,8 & $100+$ & $16,7-$ \\
\hline Individual-psicológica & 57 & $0-$ & $83,3+$ \\
\hline $\mathrm{n}$ & 51 & 6 \\
\hline
\end{tabular}

- Valor estadísticamente menor (análisis de los residuos tipificados corregidos). +Valor estadísticamente mayor (análisis de los residuos tipificados corregidos). Fuente: elaboración propia.

La tabla 4, que relaciona las aproximaciones al estudio de los encuadres con las técnicas metodológicas empleadas, permite afirmar que se produce una asociación estadísticamente significativa entre las distintas opciones $\left[\chi^{2}(1, \mathrm{n}=57)=46,58, p<0.001, v=0,90\right]$. En concreto, se confirma que el análisis de contenido es siempre empleado para llevar a cabo un acercamiento mediático y sociológico al framing $(n=51)$, mientras que la encuesta o el experimento son las herramientas usadas para abordar la aproximación individual o psicológica $(n=5)$.

Por último, acabamos el apartado de resultados abordando la condición de la teoría del framing como paradigma con estatus y entidad propios frente a otros corpus (H9), principalmente la agenda-setting. A este nivel, un 57,7 \% de los artículos examinados construyen su marco teórico apoyados en el framing, ya como única teoría (16,3\%), ya como teoría principal (41,3\%); mientras que el 31,7\% la complementan o subordinan a otros paradigmas ${ }^{5}$, finalmente, el 10,6 \% restante la emplean como teoría de background conceptual.

5 De $\operatorname{los} n=33$ papers que emplean la teoría del encuadre subordinada a otros paradigmas, en $n=21$ la vinculan a la agenda-setting; esto es, un $63,6 \%$ de ellos. 


\section{Discusión y conclusiones}

Los resultados obtenidos permiten perfilar, con relativa minuciosidad, la presencia y el uso de la teoría del encuadre en la reciente investigación hispanoamericana en comunicación. En primer lugar, la nada desdeñable cifra de 104 artículos; es decir, el 2,54 \% del total, se valen de este corpus teórico. En su inmensa mayoría, se trata de un conjunto de trabajos de corte empírico, orientados al análisis de alguna realidad fenoménica concreta relacionada con el framing. Este hecho denota un menor interés por los componentes teóricos y metodológicos del paradigma, tratados y discutidos con profusión en etapas evolutivas anteriores.

Los objetos de investigación más recurrentes son dos medios de comunicación tan arraigados históricamente como la prensa tradicional y la televisión. Este hallazgo, si bien resulta congruente con la literatura científica previa, es sorprendente debido a la menor preocupación que exhiben los autores por los denominados nuevos medios: internet, redes sociales, web 2.0, TIC, etc.; una realidad mediática de tanta actualidad que bien podrían dejar de ser etiquetados como "nuevos". En relación con los temas, y aunque sí es cierto que hay más alternancia (política, migración, guerras, medio ambiente, etc.), también llama la atención que, en el periodo estudiado (en el cual se produjo una crisis económica global aún con nítidas reminiscencias en la actualidad), no hayan proliferado más estudios acerca del tratamiento periodístico de asuntos económicos y financieros, y sus consiguientes consecuencias sociales.

Por otra parte, la investigación hispanoamericana acerca de los encuadres mediáticos se ha dirigido primordialmente a describir, de manera inductiva, la forma en que los asuntos son presentados y cubiertos en las noticias, empleando de manera hegemónica la técnica metodológica del análisis de contenido (secundada por su "antagonista" cualitativa, el análisis del discurso). No obstante, conviene aclarar que el protocolo establecido para la correcta aplicación del método ${ }^{6}$ no siempre se sigue escrupulosamente, en especial en lo concerniente al reporte de la fiabilidad intercodificadores.

6 Protocolo propuesto por expertos como Wimmer y Dominick (1996), Riffe y Freitag (1997), Neuendorf (2002) o Krippendorff(2004). 
Sin duda, esta es una carencia que convendría paliar para dotar a la investigación en análisis de contenido de un mayor rigor y transparencia, además de intentar abarcar muestras más amplias y representativas.

En cuanto a las especificidades en el tratamiento del framing, los encuadres textuales son los que suscitan un mayor interés por parte de los estudiosos. Este hallazgo, pese a ser coherente con las conclusiones a las que Matthes (2009) llega, pone de manifiesto la necesidad de compaginar el elemento textual con el visual; máxime en nuestro tiempo, en el que la imagen ha cobrado, si cabe, mayor protagonismo que antaño. Igualmente, el hecho de que la perspectiva mediática y sociológica sea la más habitual encaja con las técnicas que priman en términos metodológicos: el análisis de contenido y del discurso. Estas herramientas no permiten a los investigadores medir los efectos que los encuadres provocan en los receptores (frame effects), a saber, llegar a una óptica individual o psicológica del framing. Asimismo, los encuadres específicos, aquellos ideados en el seno de un estudio concreto y válidos casi en exclusiva para dicho estudio, eclipsan a los genéricos, los que permiten abordar distintas temáticas en diferentes contextos para hacer replicables los resultados finales.

Para terminar con este repaso sustantivo de los datos, cabe apuntar que la teoría del encuadre está adquiriendo un estatus propio que la desvincula, cada vez más, de la agenda-setting. Esta circunstancia habla de un progresivo manejo del paradigma como principal corpus teórico de los artículos en detrimento de su triangulación con otras teorías como, por caso, la citada agenda-setting, la agenda-building, el priming, la teoría del cultivo o el elaboration likelihood model (ELM) of persuasion, también presentes en la muestra.

En definitiva, son muchas las disciplinas que han incorporado, en las últimas décadas, el término framing a su jerga científica, entre ellas, además de la comunicación (de masas, política, para la salud, las relaciones públicas, etc.), la economía, la lingüística o la investigación en movimientos sociales; todas ellas expresiones de una firme convicción en el dúctil potencial explicativo de esta teoría. A la luz de las iniciativas analíticas que han centrado sus esfuerzos en sistematizar el estado de la investiga- 
ción en framing, se advierte la necesidad, "para configurarse como uno de los grandes paradigmas de la comunicación” (Sádaba, Rodríguez-Virgili y La Porte, 2008, p. 26), de seguir profundizando, no solo en su comprensión teórico-metodológica, sino, muy especialmente, en su correcta aplicación empírica. De otro modo, el paradigma verá mermados los valiosos aportes que tradicionalmente ha efectuado, y que han permitido a la comunidad científica profundizar en el conocimiento de la compleja realidad mediática circundante.

\section{Referencias}

Bateson, G. (1955). A theory of play and fantasy. Psychiatric Research Reports, 2, 39-51. Recuperado de http:/ / courses.bloodedbythought. org/play/images/7/7e/Bateson\%2C_Gregory_A_Theory_of_ Play_and_Fantasy.pdf

Borah, P. (2011). Conceptual issues in framing theory: A systematic examination of a decade's literature. Journal of Communication, 61(2), 246-263. DOI: 10.1111/j.1460-2466.2011.01539.x

Bryant, J. y Miron, D. (2004). Theory and research in mass communication. Journal of Communication, 54(4), 662-704. DOI: 10.1111/ j.1460-2466.2004.tb02650.x

Canel, M.J. (1999). El País, ABC y El Mundo: tres manchetas, tres enfoques. Zer, Revista de Estudios de Comunicación, 6, 97-117. Recuperado de https://dialnet.unirioja.es/servlet/articulo?codigo $=826304$

D’Angelo, P. (2002). News framing as a multiparadigmatic research program: A response to Entman. Journal of Communication, 52(4), 870-888. DOI: 10.1111/j.1460-2466.2002.tb02578.x

Entman, R. M. (1993). Framing: Toward clarification of a fractured paradigm. Journal of Communication, 43(4), 51-58. DOI: 10.1111/ j.1460-2466.1993.tb01304.x 
Entman, R. M., Matthes, J. y Pellicano, L. (2009). Nature, sources, and effects of news framing. En K. Wahl-Jorgensen y T. Hanitzsch (Eds.), The handbook of journalism studies (pp. 175-190). Nueva York, EE. UU.: Routledge.

Giménez-Armentia, P. (2006). Una nueva visión del proceso comunicativo: la teoría del enfoque (framing). Revista Comunicación y Hombre, 2, 55-66. Recuperado de https://dialnet.unirioja.es/servlet/ articulo? codigo $=826304$

Goffman, E. (1974). Frame analysis: An essay on the organization of experience. Cambridge, EE. UU: Harvard University Press.

Gorp, B. van (2005). Where is the frame? Victims and intruders in the Belgian press coverage of the asylum issue. European Journal of Communication, 20(4), 484-507. DOI: 10.1177/0267323105058253

Gorp, B. van (2007). The constructionist approach to framing: Bringing culture back in. Journal of Communication, 57(1), 60-78. DOI: 10.1111/j.0021-9916.2007.00329.x

Gradim, A. (2016). Framing, o enquadramento das notícias. Lisboa, Portugal: Livros Horizonte.

Hallahan, K. (1999). Seven models of framing: Implications for public relations. Journal of Public Relations Research, 11(3), 205-242. DOI: 10.1207/s1532754xjprr1103_02

Hart, P. S. (2011). The influence of episodic and thematic climate change frames on policy preferences and individual behavior chang. Science Communication, 33(1), 28-51. DOI: 10.1177/1075547010366400

Hayes, A. F. y Krippendorff, K. (2007). Answering the call for a standard reliability measure for coding data. Communication Methods and Measures, 1(1), 77-89. DOI: 10.1080/19312450709336664 
Igartua, J. J. (2006). Métodos cuantitativos de investigación en comunicación. Barcelona, España: Bosch.

Krippendorff, K. (1990). Metodología de análisis de contenido: teoría y práctica. Barcelona, España: Paidós.

Krippendorff, K. (2004). Reliability in content analysis: Some common misconceptions and recommendations. Human Communication Research, 30(3), 411-433. DOI: 10.1111/j.1468-2958.2004. tb00738.x

Martínez-Nicolás, M. y Saperas, E. (2016). Objetos de estudio y orientación metodológica de la reciente investigación sobre comunicación en España (2008-2014). Revista Latina de Comunicación Social, 71, 1365-1384. DOI: 10.4185/RLCS-2016-1150es

Matthes, J. (2009). What's in a frame? A content analysis of media framing studies in the world's leading communication journals, 1990-2005. Journalism and Mass Communication Quarterly, 86(2), 349-367. DOI: $10.1177 / 107769900908600206$

McCombs, M. y Ghanem, S. I. (2001). The convergence of agenda-setting and framing.. En S. Reese, O. Gandy y A. Grant (Eds.), Framing public life: Perspectives on media and our understanding of the social world (pp. 67-81). Mahwah, EE. UU.: Lawrence Erlbaum Associates.

McCombs, M. y Valenzuela, S. (2007). The agenda-setting theory. Cuadernos.info, 20, 44-50. Recuperado de https://dialnet.unirioja.es/servlet/articulo? codigo $=826304$

Muñiz, C., Igartua, J. J., Fuente, M. de la y Otero, J. A. (2008). Imágenes periodísticas de la inmigración: aportaciones metodológicas al estudio de la comunicación visual. Anàlisi, 37, 31-48. Recuperado de https://core.ac.uk/download/pdf/13275978.pdf 
Neuendorf, K. A. (2002). The content analysis guidebook. Thousand Oaks, EE. UU.: Sage.

Reese, S. D. (2001). A bringing model for media research. En S. Reese, O. Gandy y A. Grant (Eds.), Framing public life: Perspectives on media and our understanding of the social world (pp. 7-31). Mahwah, EE. UU.: Lawrence Erlbaum Associates.

Riffe, D.y Freitag, A. (1997). A content analysis of content analyses: Twenty-five years of Journalism Quarterly. Journalism and Mass Communication Quarterly, 74(4), 873-882. DOI: 10.1177/107769909707400306

Sádaba Garraza, T. (2001). Origen, aplicación y límites de la "teoría del encuadre" (framing) en comunicación. Comunicación y Sociedad, 14(2), 143-175. Recuperado de https://www.unav.es/fcom/communication-society/es/articulo.php?art_id=335

Sádaba Garraza, T., La Porte Fernández-Alfaro, M. T. y Rodríguez Virgili, J. (2008). La teoría del framing en la investigación en comunicación política. En M.J. Canel Crespo y M. García Gurrionero (Coords.), Estudios de comunicación política: libro del año 2008 (pp. 15-30). Madrid: Asociación de Comunicación Política.

Saperas, E. y Carrasco-Campos, Á. (2015). The operationalization of the concept of framing in the Journal of Communication (2009-2013): Objects of study, research techniques and theoretical construction. Communication \& Society, 28(4), 49-66. DOI: 10.15581/003.28.4.49-66

Scheufele, D. A. (1999). Framing as a theory of media effects. Journal of Communication, 49(1), 103-122.DOI: 10.1111/j.1460-2466.1999. tb02784.x

Scheufele, D. A. y Tewksbury, D. (2007). Framing, agenda-setting, and priming: The evolution of three media effects models. Journal of Communication, 57(1), 9-20. DOI: 10.1111/j.0021-9916.2007.00326.x 
Semetko, H. A. y Valkenburg, P. M. (2000). Framing European Politics: A content analysis of press and television news. Journal of Communication, 50(2), 93-109. DOI: 10.1111/j.1460-2466.2000.tb02843.x

Vicente-Mariño, M. y López-Rabadán, P. (2009). Resultados actuales de la investigación sobre framing: sólido avance internacional y arranque de la especialidad en España. Zer, Revista de Estudios de Comunicación, 14(26), 13-34. Recuperado de http://www.ehu.eus/ ojs/index.php/Zer/article/view/2750

Vliegenthart, R. y Zoonen, L. van (2011). Power to the frame: Bringing sociology back to frame analysis. European Journal of Communication, 26(2), 101-115. DOI: $10.1177 / 0267323111404838$

Vreese, C. H. de (2005). News framing: Theory and typology. Information Design Journal, 13(1), 51-62. DOI: 10.1075/idjdd.13.1.06vre

Vreese, C. H. de, Peter, J. y Semetko, H. A. (2001). Framing politics at the launch of the euro: A cross-national comparative study of frames in the news. Political Communication, 18(2), 107-122. DOI: $10.1080 / 105846001750322934$

Weaver, D. H. (2007). Thoughts on agenda-setting, framing, and priming. Journal of Communication, 57(1), 142-147. DOI: 10.1111/j.14602466.2006.00333.x

Wimmer, R. D. y Dominick, J. R. (1996). La investigación cientifica de los medios de comunicación: una introducción a sus métodos. Barcelona, España: Bosch. 


\section{Anexo}

\section{Fiabilidad de las variables del libro de códigos}

\begin{tabular}{|l|c|}
\hline \multicolumn{1}{|c|}{ Variable } & Alpha de Krippendorff \\
\hline 1. Objeto de la investigación & 0,92 \\
\hline 2. Temática del estudio & 1 \\
\hline 3. Modalidad del estudio & 0,83 \\
\hline 4. Tipo de estudio & 1 \\
\hline 5. Técnicas metodológicas empleadas & 0,88 \\
\hline 5.1. Reporte de fiabilidad intercodificadores & 0,86 \\
\hline 6. Muestra de casos o individuos & 0,78 \\
\hline 7.1. Especificidad de estudio (i) & 0,74 \\
\hline 7.2. Especificidad de estudio (ii) & 0,72 \\
\hline 7.3. Especificidad de estudio (iii) & 0,74 \\
\hline 8. Construcción teórica de la investigación & 0,78 \\
\hline Media total & 0,84 \\
\hline
\end{tabular}

Fuente: elaboración propia. 